\title{
SEPARATE AND JOINT PROPERTIES OF SOME ANALOGUES OF POINTWISE DISCONTINUITY
}

\author{
VASYL' NESTERENKO
}

\begin{abstract}
We study separate and joint properties of pointwise discontinuity, simple continuity and mild continuity of functions of two variables. In particular, it is shown that for a Baire space $X$, a Baire space $Y$ which has a countable pseudobase and for a metric space $Z$, a function $f: X \times Y \rightarrow Z$ is pointwise discontinuous if and only if $f$ satisfies $(\alpha, \beta)$-condition and condition (C), and $M=\{x \in X$ : $\left.\overline{C\left(f^{x}\right)}=Y\right\}$ is a residual subset of $X$. In addition, a characterization of simple continuity for mappings of one and two variables is given.
\end{abstract}

\section{Introduction}

A mapping $f: X \rightarrow Y$ is called pointwise discontinuous if the set $C(f)$ of points of continuity of $f$ is dense in $X$. There are many analogues of pointwise discontinuity (cliquish [1] quasi-continuity [2], simple continuity [3], mild continuity [4, etc.) that are investigated in the works of many mathematicians.

Among many problems for mappings of several variables, problems related to their separate and joint properties occupy a special place. The investigation of relationships between separate and joint continuity, which started its history in classical works of Baire and Osgood, has spread out on various weakening of continuity. In particular, in [5], the relationship between different separate and joint properties of weakening of continuity (quasi-continuity, almost continuity, somewhat continuity, etc.) is investigated. In [6]-8], separate properties of functions $f: X \times Y \rightarrow \mathbb{R}$, for which sections $f_{y}=f(\cdot, y), y \in Y$ are monotone, lower and upper quasi-continuous or have closed graph, have been studied.

For the mapping $f: X \times Y \rightarrow Z$ of two variables, it is an interesting problem to find minimum conditions for sections

$$
f^{x}=f(x, \cdot), x \in X \quad \text { and } \quad f_{y}=f(\cdot, y), y \in Y
$$

(c) 2014 Mathematical Institute, Slovak Academy of Sciences.

2010 Mathematics Subject Classification: Primary 54C08, 26B05; Secondary 54C30, 26A15.

Keyw ords: simple continuity, mild continuity, cliquishness, pointwise discontinuity. 


\section{VASYL' NESTERENKO}

at which the mapping $f$ has a property of some weakened continuity. In [2], S. K e m pisty proved that a separately quasi-continuous function $f: \mathbb{R}^{n} \rightarrow \mathbb{R}$ is jointly quasi-continuous. The Kempisty's result was generalized by many mathematicians. In 9, the following characterization for quasicontinuity mappings of two variables was obtained: if $X$ is a Baire space, $Y$ a space which has a countable base, and $Z$ a separable metrizable space, then a mapping $f: X \times Y \rightarrow Z$ is quasi-continuous if and only if $f$ is horizontally quasi-continuous, and $\left\{x \in X: f^{x}\right.$ is quasi-continuous $\}$ is a residual subset of $X$.

In contrast to quasi-continuity, cliquishness or pointwise discontinuity relative to each variable does not guarantee jointly cliquishness or more pointwise discontinuity. For cliquishness, L. F u d a li did the first attempt to characterize this concept for functions of two variables. In [10, he showed that if $X$ is a Baire space, $Y$ a space which has a countable pseudo-base, $Z$ a metric space, and if a function $f: X \times Y \rightarrow Z$ is such that sections $f_{y}$ are quasi-continuous for each $y \in Y$ and sections $f^{x}$ are cliquish for each $x \in X$, then $f$ is joint cliquish. In [11], the following characterization of cliquishness for functions of two variables is obtained: if $X$ is a Baire space, $Y$ a space which has a countable pseudo-base, $Z$ a metric space, then the function $f: X \times Y \rightarrow Z$ is cliquish if and only if $f$ satisfies $(\alpha, \beta)$-condition and condition (C), and $M=\left\{x \in X: f^{x}\right.$ is cliquish $\}$ is a residual subset of $X$.

In [12, for pointwise discontinuity, the following result was obtained: if $X$ is a Baire space, $Y$ a Baire space which has a countable pseudo-base, $Z$ a metrizable space, a mapping $f X \times Y \rightarrow Z$ is horizontally quasi-continuous, and $f^{x}$ is pointwise discontinuous for each $x \in X$, then

$$
A=\{x \in X: \overline{\{y \in Y:(x, y) \in C(f)\}}=Y\}
$$

is a residual subset of $X$, and if $X \times Y$ is a Baire space, then $f$ is pointwise discontinuous.

In this article, for functions of two variables, we obtain a characterization of pointwise discontinuity, we establish sufficient conditions for simple continuity and mild continuity as well as we obtain new characterizations of simple continuity for mappings of one and two variables.

\section{Basic definitions and concepts}

Let $(Z, d)$ be a metric space. Recall that for a non-empty subset $A$ of a set $X$ and for a function $g: T \rightarrow Z$, the number $\omega_{g}(A)=\sup \left\{d\left(g\left(x^{\prime}\right), g\left(x^{\prime \prime}\right)\right): x^{\prime}, x^{\prime \prime} \in A\right\}$ is called the oscillation of $g$ on the set $A$. If $T$ is a topological space and $\mathcal{U}_{t}$ is a system of neighborhoods of $t$ in $T$, then the number $\omega_{g}(t)=\inf \left\{\omega_{g}(U): U \in \mathcal{U}_{t}\right\}$ is called the oscillation of $g$ at $t$. Obviously, $t \in C(g)$ if and only if $\omega_{g}(t)=0$. 


\section{SEPARATE AND JOINT PROPERTIES OF POINTWISE DISCONTINUITY}

For a subset $A$ of a topological space, let int $A$ and $\bar{A}$ denote the interior and the closure of $A$, respectively.

Let $Y$ be a metric space. A function $f: X \rightarrow Y$ is said to be cliquish at a point $x \in X[1]$ if for any $\varepsilon>0$ and a neighborhood $U$ of $x \in X$ there exists a nonempty open subset $G$ of $X$ such that $G \subseteq U$ and $\omega_{f}(G)<\varepsilon$, and cliquish, if it is such at any point. Obviously, each pointwise discontinuous function with values in a metric space is cliquish. It is well-known ([1]) that if $X$ is a Baire space, then each cliquish function $f: X \rightarrow Y$ is pointwise discontinuous.

Let $Y$ be now a topological space. A map $f: X \rightarrow Y$ is said to be quasi-continuous at $x \in X$ [13] if for each neighborhood $V$ of $y=f(x)$ in $Y$ and each neighborhood $U$ of $x$ in $X$, there is a nonempty open subset $G$ of $X$ such that $G \subseteq U$ and $f(G) \subseteq V$. A map $f: X \rightarrow Y$ is said to be quasicontinuous if it is such at each point of the space $X$. It is easy to see that every quasi-continuous mapping with values in a metric space is cliquish. The converse is not true.

A mapping $f: X \rightarrow Y$ is simply continuous [3] if, for each open subset $V$ of $Y$, the set $f^{-1}(V)$ is the union of an open subset $U$ of $X$ and a nowhere dense subset $N$ of $X$. The last condition is equivalent to the fact that the set $f r\left(f^{-1}(V)\right)$ is nowhere dense, i.e., int $\overline{f r\left(f^{-1}(V)\right)}=\varnothing$ where $f r(A)$ is a boundary of the set $A$.

Put $S_{f}=\{x \in X$ there is a base $\mathcal{A}$ of neighbourhoods of $f(x)$ such that for each $A \in \mathcal{A}$ and each neighbourhood $U$ of $x, f^{-1}(A) \backslash i n t f^{-1}(A)$ is not dense in $U\}$. If the set $S_{f}$ is dense in $X$, then $f$ is called mildly continuous [4]. A function $f$ is called almost continuous (in the sense of Husain) at $x \in X$ [14] if, for each neighborhood $V$ of $y=f(x)$ in $Y$, there is a subset $A$ of $X$ such that $x \in \operatorname{int} \bar{A}$ and $f(A) \subseteq V$. Let $Q(f)$ and $P(f)$ denote the sets of all points at which the mapping $f$ is quasi-continuous and almost continuous, respectively. In [15, Theorem 2.3], it is shown that $S_{f}=(X \backslash P(f)) \cup Q(f)$. It is easy to see that a quasi-continuous mapping is mildly continuous. In [4, Lemma 2], it is shown that a cliquish function is mildly continuous.

\section{Some properties of simple continuity}

The following characterization of quasi-continuity is well-known (see [16, Proposition 3.1.1]).

TheOREM 3.1. Let $X$ and $Y$ be topological spaces. $A$ mapping $f: X \rightarrow Y$ is quasicontinuous if and only if for any nonempty open subset $U$ of $X$ and for any subset $A$ of $X$ such that $U \subseteq \bar{A}$, we have $f(U) \subseteq \overline{f(A)}$.

Simple continuity has a similar characterization.

Theorem 3.2. Let $X$ and $Y$ be topological spaces. A mapping $f: X \rightarrow Y$ is simply continuous if and only if the following condition holds 
( $\star$ for any nonempty open subset $U$ of $X$ and any subset $A$ of $X$ with $U \subseteq \bar{A}$, there exists a nonempty open subset $G$ of $X$ such that

$$
G \subseteq U \text { and } f(G) \subseteq \overline{f(A)} .
$$

Proof. Necessity. Let the mapping $f$ be simply continuous but do not let it satisfy condition $(\star)$. Then, there exist a nonempty open subset $U$ of $X$ and a set $E$ dense in $U$ such that for each nonempty open subset $G \subseteq U$ there is a point $x_{G} \in G$ such that $f\left(x_{G}\right) \notin \overline{f(E)}$. Consider

the open set $V=Y \backslash \overline{f(E)}$ and the set $A=\left\{x_{G}: G\right.$ is an open subset of $\left.U\right\}$.

It is clear that

$$
\bar{A} \supseteq U \text { and } E \cap A=\varnothing .
$$

Since the mapping $f$ is simply continuous, $f r\left(f^{-1}(V)\right)=\overline{f^{-1}(V)} \cap \overline{X \backslash f^{-1}(V)}$ is a nowhere dense set. However,

$$
f^{-1}(V) \supseteq A \text { and therefore, } \overline{f^{-1}(V)} \supseteq \bar{A} \supseteq U .
$$

In addition,

$$
X \backslash f^{-1}(V) \supseteq E \quad \text { and } \quad \overline{X \backslash f^{-1}(V)} \supseteq \bar{E} \supseteq U
$$

Hence,

$$
f r\left(f^{-1}(V)\right) \supseteq U \neq \varnothing,
$$

what is impossible because the set $f r\left(f^{-1}(V)\right)$ is nowhere dense. Thus, the assumption is not true.

Sufficiency. Let the mapping $f$ satisfy condition $(\star)$ but do not let it be simply continuous. Since $f$ is not simply continuous, there is a nonempty open subset $V$ of $Y$ such that the set $f r\left(f^{-1}(V)\right)$ is dense in some nonempty open subset $U$ of $X$. Then,

Since,

$$
f r\left(f^{-1}(V)\right)=\overline{f r\left(f^{-1}(V)\right)} \supseteq U
$$

$$
f r\left(f^{-1}(V)\right)=\overline{f^{-1}(V)} \cap \overline{X \backslash f^{-1}(V)}, \quad \overline{f^{-1}(V)} \supseteq U \quad \text { and } \quad \overline{X \backslash f^{-1}(V)} \supseteq U .
$$

From condition $(\star)$, it follows that there is a nonempty open subset $G$ of $X$ such that

Then,

$$
G \subseteq U \quad \text { and } \quad f(G) \subseteq \overline{f\left(X \backslash f^{-1}(V)\right)} .
$$

$$
f(G) \subseteq \overline{f\left(X \backslash f^{-1}(V)\right)} \subseteq \overline{Y \backslash V}=Y \backslash V .
$$

On the other hand,

$$
f(G) \cap V \neq \varnothing, \quad \text { because } \overline{f^{-1}(V)} \supseteq U \text { and } \quad G \subseteq U .
$$

The obtained contradiction completes the proof of sufficiency. 


\section{SEPARATE AND JOINT PROPERTIES OF POINTWISE DISCONTINUITY}

The next result follows easily from Theorem 3.1 and Theorem 3.2.

Corollary 3.2.1. Let $X$ and $Y$ be topological spaces and $f: X \rightarrow Y$ a quasi-continuous mapping. Then, $f$ is simply continuous.

It is possible to get a more accurate result.

ThEOREM 3.3. Let $X$ and $Y$ be topological spaces, $f: X \rightarrow Y$ a mapping, and $X \backslash Q(f)$ a nowhere dense subset of $X$. Then, $f$ is simply continuous.

P r o o f. Consider a nonempty open subset $U$ of $X$ and a subset $E$ of $X$ such that $U \subseteq \bar{E}$. Since $X \backslash Q(f)$ is a nowhere dense set, there is a nonempty open subset $G$ of $X$ such that $G \subseteq U \cap Q(f)$. Put $g=\left.f\right|_{G}$. The mapping $g$ is quasi-continuous at each point of $G$. Since $E$ is dense in $U, \overline{E \cap G} \supseteq G$. From Theorem 3.1, it follows that

$$
f(G)=g(G) \subseteq \overline{g(E \cap G)}=\overline{f(E \cap G)} \subseteq \overline{f(E)} .
$$

Hence, $f$ is simply continuous.

Note that Theorem 3.3 follows immediately from [17, Lemma 1.1].

The converse is not true. It is shown in the following example from [18, Example 1].

ExAmple 1. Let $\mathbb{Q}=\left\{r_{n}: n \in \mathbb{N}\right\}$ be a set of rational numbers. Let the function $f: \mathbb{R} \rightarrow \mathbb{R}$ be given by

$$
f(x)= \begin{cases}x+\frac{1}{n}, & x=r_{n}, \\ x, & \text { otherwise }\end{cases}
$$

The function $f$ is simply continuous, and $\mathbb{R} \backslash Q(f)=\mathbb{Q}$.

\section{Jointly pointwise discontinuity}

Let $Z$ be a metric space. We say that a function $f: X \times Y \rightarrow Z$ satisfies $(\alpha, \beta)$-condition if for any $\alpha, \beta$ with $0<\alpha<\beta$, any nonempty open subsets $U$ of $X$ and $V$ of $Y$ and for any set $E \subseteq X$ dense in $U$ with $\omega_{f}(E \times V)<\alpha$, there exist nonempty open subsets $G$ of $X$ and $H$ of $Y$ such that $G \subseteq U, H \subseteq V$ and $\omega_{f}(G \times H)<\beta$. A function $f: X \times Y \rightarrow Z$ satisfies condition $(C)$ if for any $\varepsilon>0$, any non-meager subset $E$ of $X$ and any nonempty open subset $V$ of $Y$, there exist a somewhere dense subset $E_{1}$ of $X$ and a mapping $g: E_{1} \rightarrow V$ such that $E_{1} \subseteq E$ and $\omega_{f}(G r(g))<\varepsilon$, where $\operatorname{Gr}(g)=\left\{(x, g(x)): x \in E_{1}\right\}$ is the graph of $g$.

Note that if $Z$ is a separable metric space, then an arbitrary function $f: X \times Y \rightarrow Z$ satisfies condition (C). Also, notice that if a function $f: X \times Y \rightarrow Z$ is simply continuous, then it satisfies $(\alpha, \beta)$-condition. 
Put

$$
C^{x}(f)=\{y \in Y:(x, y) \in C(f)\}
$$

Theorem 4.1. Let $X$ be a topological space, $Y$ a topological space which has a countable pseudo-base, $(Z, d)$ a metric space, $f: X \times Y \rightarrow Z$ a function such that $(\alpha, \beta)$-condition and condition $(C)$ hold, and let $M=\left\{x \in X: \overline{C\left(f^{x}\right)}=Y\right\}$ be a residual subset of $X$. Then, $A=\left\{x \in X: C^{x}(f)\right.$ is a residual subset of $\left.Y\right\}$ is a residual subset of $X$.

P r o o f. Let $\left\{V_{n}: n \in \mathbb{N}\right\}$ be a pseudo-base of $Y$. Assume the contrary. Let $X \backslash A$ be a non-meager subset of $X$. Put $B=(X \backslash A) \cap M$. It is clear that $B$ is also a non-meager subset of $X$. For each $x \in B$, there is a non-meager subset $W_{x}$ of $Y$ such that $f$ is discontinuous at each point of $\{x\} \times W_{x}$. Then, $W_{x}=\bigcup_{k=1}^{\infty} W_{x, k}$, where $W_{x, k}=\left\{y \in W_{x}: \omega_{f}(x, y) \geq \frac{1}{k}\right\}$. Since $W_{x}$ is a non-meager subset of $Y$ for each $x \in B$, there are positive integers $j_{x}$ and $k_{x}$ such that $\overline{W_{x, k_{x}}} \supseteq V_{j_{x}}$. Consider sets $A_{j, k}=\left\{x \in B: j_{x}=j, k_{x}=k\right\}$. Since $B=\bigcup_{j, k=1}^{\infty} A_{j, k}$, there are positive integers $m$ and $n$ such that $A_{m, n}$ is a non-meager subset of $X$.

Let $\left\{V_{m, i}: i \in \mathbb{N}\right\}$ be a pseudo-base subspace of $V_{m}$ of $Y$. Consider the sets $B_{i}=\left\{x \in A_{m, n}: \omega_{f}\left(\{x\} \times V_{m, i}\right)<\frac{1}{8 n}\right\}$. Since for each $x \in A_{m, n} \subseteq B$ the function $f^{x}$ is pointwise discontinuous, $A_{m, n}=\bigcup_{i=1}^{\infty} B_{i}$. Hence, there is a positive integer $l$ such that $B_{l}$ is a non-meager subset of $X$.

Put $V=V_{m, l}$. Since $f$ satisfies condition (C), there are a somewhere dense subset $E$ of $X$ and the mapping $g: E \rightarrow V$ such that $E \subseteq B_{l}$ and $\omega_{f}(G r(g))<\frac{1}{8 n}$. The open set $U=i n t \bar{E}$ is nonempty because the set $E$ is somewhere dense. We show that $\omega_{f}(E \times V) \leq \frac{3}{8 n}$. Take the points $p_{i}=\left(u_{i}, v_{i}\right) \in E \times V, i=1,2$. Then,

$$
\begin{aligned}
d\left(f\left(p_{1}\right), f\left(p_{2}\right)\right) \leq & d\left(f\left(p_{1}\right), f\left(u_{1}, g\left(u_{1}\right)\right)\right) \\
& +d\left(f\left(u_{1}, g\left(u_{1}\right)\right), f\left(u_{2}, g\left(u_{2}\right)\right)\right) \\
& +d\left(f\left(u_{2}, g\left(u_{2}\right)\right), f\left(p_{2}\right)\right) \\
\leq & \frac{1}{8 n}+\frac{1}{8 n}+\frac{1}{8 n}=\frac{3}{8 n} .
\end{aligned}
$$

Hence, $\omega_{f}(E \times V) \leq \frac{3}{8 n}<\frac{1}{2 n}$.

Since $f$ satisfies $(\alpha, \beta)$-condition, for $\alpha=\frac{1}{2 n}$ and $\beta=\frac{1}{n}$, there are nonempty open subsets $G$ of $X$ and $H$ of $Y$ such that $G \subseteq U, H \subseteq V$ and $\omega_{f}(G \times H)<\frac{1}{n}$. Take any point $a \in G \cap B_{l}$. Since $B_{l} \subseteq A_{m, n}$ and for each $x \in A_{m, n}$, we have that

$$
H \subseteq V=V_{m, l} \subseteq V_{m} \subseteq \overline{W_{x, n}},
$$




\section{SEPARATE AND JOINT PROPERTIES OF POINTWISE DISCONTINUITY}

the set $H \cap W_{a, n}$ is nonempty. Take any point $b \in H \cap W_{a, n}$. The set $G \times H$ is a neighborhood of $(a, b)$. Then, both $\omega_{f}(a, b)<\frac{1}{n}$ and $\omega_{f}(a, b) \geq \frac{1}{n}$. This contradiction proves the theorem.

Corollary 4.1.1. Let $X$ be a Baire space, $Y$ a Baire space which has a countable pseudo-base, $Z$ a metric space, $f: X \times Y \rightarrow Z$ a function such that $(\alpha, \beta)$-condition and condition $(C)$ hold, and let $M=\left\{x \in X: \overline{C\left(f^{x}\right)}=Y\right\}$ be a residual subset of $X$. Then, $f$ is pointwise discontinuous.

P r o of. Since a residual subset of a Baire space is everywhere dense, by Theorem 4.1, $A=\left\{x \in X: C^{x}(f)\right.$ is a residual subset of $\left.Y\right\}$ is a dense subset of $X$. In addition,

Therefore,

$$
A \subseteq\left\{x \in X: \overline{C^{x}(f)}=Y\right\} \text { and } \bigcup_{x \in A}\left(\{x\} \times C^{x}(f)\right) \subseteq C(f) .
$$

$$
\overline{\bigcup_{x \in A}\left(\{x\} \times C^{x}(f)\right)}=X \times Y \text { and } \overline{C(f)}=X \times Y .
$$

Theorem 4.2. Let $X$ be a topological space, $Y$ a Baire space which has a countable pseudo-base, $Z$ a metric space and $f: X \times Y \rightarrow Z$ a pointwise discontinuous function. Then, $f$ satisfies $(\alpha, \beta)$-condition and condition $(C)$, and $M=\{x \in$ $\left.X: \overline{C\left(f^{x}\right)}=Y\right\}$ is a residual subset of $X$.

P r o o f. First, we show that $f$ satisfies $(\alpha, \beta)$-condition. Take arbitrary numbers $0<\alpha<\beta$, nonempty open subsets $U$ of $X$ and $V$ of $Y$, and a subset $E$ of $X$ with $U \subseteq \bar{E}$ such that $\omega_{f}(E \times V)<\alpha$. Since $f$ is pointwise discontinuous, $C(f) \cap(U \times V) \neq \varnothing$. Take the point $p \in C(f) \cap(U \times V)$. By continuity of $f$ at $p$, it follows that there are nonempty open subset $G$ of $X$ and $H$ of $Y$ such that $G \subseteq U, H \subseteq V$ and $\omega_{f}(G \times H)<\beta$.

We show that $f$ satisfies condition $(\mathrm{C})$. Take any $\varepsilon>0$, any non-meager subset $E$ of $X$ and any nonempty open subset $V$ of $Y$. Since $E$ is a non-meager set, int $\bar{E} \neq \varnothing$. Put $U=$ int $\bar{E}$. Since $f$ is pointwise discontinuous, $C(f) \cap(U \times V) \neq \varnothing$. Take the point $p=(a, b) \in C(f) \cap(U \times V)$. By continuity of $f$ at $p$, it follows that there are nonempty open subset $G$ of $X$ and $H$ of $Y$ such that $a \in G \subseteq U$, $b \in H \subseteq V$ and $\omega_{f}(G \times H)<\varepsilon$. Put $E_{1}=E \cap G$. Then, int $\overline{E_{1}} \neq \varnothing$. Consider the mapping $g: E_{1} \rightarrow V, g(x)=b$ for all $x \in E_{1}$. Then, $\omega_{f}(G r(g)) \leq \omega_{f}(G \times H)<\varepsilon$.

Now, we show that $M=\left\{x \in X: \overline{C\left(f^{x}\right)}=Y\right\}$ is residual subset of $X$. Let $\left\{V_{n}: n \in \mathbb{N}\right\}$ be a pseudo-base of $Y$. Assume the contrary. Let $E=X \backslash M$ be a non-meager subset of $X$. For each $x \in E$, there is a nonempty open subset $W_{x}$ of $Y$ such that $W_{x} \cap C\left(f^{x}\right)=\varnothing$. The sets $W_{x, k}=\left\{y \in W_{x}: \omega_{f^{x}}(y) \geq \frac{1}{k}\right\}$ are closed in $W_{x}$ and $W_{x}=\bigcup_{k=1}^{\infty} W_{x, k}$. Since $W_{x}$ is non-meager subset of $Y$, for each $x \in E$, there are positive integers $j_{x}$ and $k_{x}$ such that $W_{x, k_{x}} \supseteq V_{j_{x}}$. 
Consider the sets $A_{j, k}=\left\{x \in E: j_{x}=j, k_{x}=k\right\}$. Since $E=\bigcup_{j, k=1}^{\infty} A_{j, k}$, there are positive integers $m$ and $n$ such that the set $A_{m, n}$ is dense in some nonempty open subset $U$ of $X$. By pointwise discontinuity of $f$, it follows that $C(f) \cap\left(U \times V_{m}\right) \neq \varnothing$. Take any point $p \in C(f) \cap\left(U \times V_{m}\right)$. Then, there are nonempty subsets $G$ of $X$ and $H$ of $Y$ such that $G \subseteq U, H \subseteq V_{m}$ and $\omega_{f}(G \times H)<\frac{1}{n}$. Take any points $a \in G \cap A_{m, n}$ and $b \in H \cap W_{a, n}$. Then, both $\omega_{f^{a}}(b) \geq \frac{1}{n}$ and $\omega_{f^{a}}(b) \leq \omega_{f}(G \times H)<\frac{1}{n}$. This contradiction proves the theorem.

Corollary 4.2.1. Let $X$ be a Baire space, $Y$ a Baire space which has a countable pseudo-base, $Z$ a metric space. A function $f: X \times Y \rightarrow Z$ is pointwise discontinuous if and only if $f$ satisfies $(\alpha, \beta)$-condition and condition $(C)$, and $M=\left\{x \in X: \overline{C\left(f^{x}\right)}=Y\right\}$ is a residual subset of $X$.

\section{Jointly simple continuity}

TheOrem 5.1. Let $X$ be a Baire space, $Y$ a Baire space which has a countable pseudo-base and $Z$ a separable metrizable space. A mapping $f: X \times Y \rightarrow Z$ is simply continuous if and only if the following condition holds:

( $\star \star)$ for each nonempty open subsets $U$ of $X$ and $V$ of $Y$, a non-meager subset $M$ of $X$ with $M \subseteq U$ and subsets $B_{x}, x \in M$ of $Y$ with $V \subseteq \overline{B_{x}}$, there are nonempty open subsets $G$ of $X$ and $H$ of $Y$ such that

$$
G \times H \subseteq i n t \bar{M} \times V \quad \text { and } \quad f(G \times H) \subseteq \overline{f\left(\bigcup_{x \in M}\left(\{x\} \times B_{x}\right)\right)} .
$$

P r o o f. We will use Theorem 3.2 to characterize the notion of simple continuity. Since $Z$ is a separable metrizable space, $Z$ is a regular second countable space. Let $\left\{V_{n}: n \in \mathbb{N}\right\}$ be a pseudo-base of $Y$ and $\left\{W_{k}: k \in \mathbb{N}\right\}$ a base of $Z$.

The proof of necessity is obvious because the set $\bigcup_{x \in M}\left(\{x\} \times B_{x}\right)$ is dense in $i n t \bar{M} \times V$.

Sufficiency will be proved by contradiction. Suppose $f$ is not simply continuous. Then, there are nonempty open subsets $U$ of $X$ and $V$ of $Y$ and a subset $E$ of $X \times Y$ with $U \times V \subseteq \bar{E}$ such that $f(G \times H) \not \subset \overline{f(E)}$ for any nonempty open subsets $G$ of $X$ and $H$ of $Y$ with $G \times H \subseteq U \times V$.

Consider the set

$$
A=\left\{x \in U:\left(\exists B_{x} \subseteq V, V \backslash B_{x} \text { is a meager set }\right)\left(\forall y \in B_{x}\right)(f(x, y) \notin \overline{f(E)})\right\} .
$$




\section{SEPARATE AND JOINT PROPERTIES OF POINTWISE DISCONTINUITY}

We show that $A$ is a non-meager set. Suppose not. Let $A$ be a meager set. Since $X$ is a Baire space, $U \backslash A$ is a non-meager set. For each $n \in \mathbb{N}$, we consider the sets

$$
A_{n}=\left\{x \in U \backslash A:\left(\exists D_{x} \subseteq V, \overline{D_{x}} \supseteq V_{n}\right)\left(\forall y \in D_{x}\right)(f(x, y) \in \overline{f(E)})\right\} .
$$

We show that $U \backslash A \subseteq \bigcup_{n=1}^{\infty} A_{n}$. Let $x \in U \backslash A$. Since $x \notin A$, for each set $B_{x} \subseteq V$ such that $V \backslash B_{x}$ is a meager set, there is a point $y \in B_{x}$ such that $f(x, y) \in \overline{f(E)}$. Put

$$
D_{x}=\left\{y \in V:\left(\exists B_{x} \subseteq V, V \backslash B_{x} \text { is a meager set }\right)\left(y \in B_{x}\right)(f(x, y) \in \overline{f(E)})\right\} .
$$

It is clear that the set $D_{x}$ is non-meager. Then, there is a positive integer $n$ such that $\overline{D_{x}} \supseteq V_{n}$. Hence, $x \in A_{n}$ and $U \backslash A \subseteq \bigcup_{n=1}^{\infty} A_{n}$.

Since $U \backslash A$ is a non-meager set, there is a positive integer $k$ such that $A_{k}$ is a non-meager set. By condition $(\star \star)$, there are nonempty open subsets $G$ of $X$ and $H$ of $Y$ such that Then,

$$
G \times H \subseteq i n t \overline{A_{k}} \times V_{k} \subseteq U \times V \text { and } f(G \times H) \subseteq \overline{f\left(\bigcup_{x \in A_{k}}\left(\{x\} \times D_{x}\right)\right)}
$$

$$
f(G \times H) \subseteq \overline{f\left(\bigcup_{x \in A_{k}}\left(\{x\} \times D_{x}\right)\right)} \subseteq \overline{\overline{f(E)}}=\overline{f(E)} .
$$

This contradiction proves that $A$ is a non-meager set.

Since $Y$ is a Baire space, for each $x \in A$, the set $B_{x}$ is non-meager. For any $x \in A$, there are positive integers $n_{x}$ and $m_{x}$ such that for each $y \in B_{x} \cap V_{n_{x}}$ we have $f(x, y) \in W_{m_{x}}$ and $\overline{W_{m_{x}}} \cap f(E)=\varnothing$. Consider the sets $A_{n, m}=\{x \in A$ : $\left.n_{x}=n, m_{x}=m\right\}$. It is clear that $A=\bigcup_{m, n=1}^{\infty} A_{n, m}$. Since $A$ is a non-meager set, there are positive integers $n_{0}$ and $m_{0}$ such that $A_{n_{0}, m_{0}}$ is a non-meager set.

Now again, we apply condition $(\star \star)$. Then, there are nonempty open subsets $G$ of $X$ and $H$ of $Y$ such that

$$
G \times H \subseteq \operatorname{int} \overline{A_{n_{0}, m_{0}}} \times V_{n_{0}} \subseteq U \times V
$$

and

$$
f(G \times H) \subseteq f \overline{\left(\bigcup_{x \in A_{n_{0}, m_{0}}}\left(\{x\} \times\left(B_{x} \cap V_{n_{0}}\right)\right)\right)} .
$$

Therefore,

$$
f(G \times H) \subseteq \overline{f\left(\bigcup_{x \in A_{n_{0}, m_{0}}}\left(\{x\} \times\left(B_{x} \cap V_{n_{0}}\right)\right)\right)} \subseteq \overline{W_{m_{0}}} .
$$

Since $\overline{W_{m_{0}}} \cap f(E)=\varnothing, f(G \times H) \cap f(E)=\varnothing$. But it contradicts the fact that $U \times V \subseteq \bar{E}$. This contradiction proves that $f$ is simply continuous. 
A mapping $f: X \times Y \rightarrow Z$ satisfies condition $(B)$ if for each nonempty open subsets $U$ of $X$ and $V$ of $Y$ and a subset $A$ of $X$ with $U \subseteq \bar{A}$, there are nonempty open subsets $G$ of $X$ and $H$ of $Y$ such that $G \times H \subseteq U \times V$ and $f(G \times H) \subseteq \overline{f(A \times V)}$.

We note that horizontally quasi-continuous ([19]) or simply continuous mappings satisfy condition (B). For a metric space $Z$, if a function $f: X \times Y \rightarrow Z$ satisfies condition $(\mathrm{B})$ then $f$ satisfies $(\alpha, \beta)$-condition.

Theorem 5.2. Let $X$ be a Baire space, $Y$ a Baire space which has a countable pseudo-base and $Z$ a separable metrizable space, $f: X \times Y \rightarrow Z$ a function such that condition $(B)$ holds, and $E=\left\{x \in X: f^{x}\right.$ is simply continuous $\}$ a residual subset of $X$. Then, $f$ is simply continuous.

P r o o f. We will use Theorem 5.1. Take arbitrary nonempty open subsets $U$ of $X$ and $V$ of $Y$ and a non-meager subset $M$ of $X$ such that $M \subseteq U$. For each $x \in M$, we consider a subset $B_{x}$ of $Y$ such that $V \subseteq \overline{B_{x}}$. Let $\left\{V_{n}: n \in \mathbb{N}\right\}$ be a pseudobase of $Y$. For each $n \in \mathbb{N}$, we consider the set

$$
A_{n}=\left\{x \in M \cap E: f^{x}\left(V_{n}\right) \subseteq \overline{f^{x}\left(B_{x}\right)}\right\} .
$$

Note that $M \cap E$ is a non-meager set. For each $x \in M$, the mapping $f^{x}$ is simply continuous. Therefore, $\bigcup_{n=1}^{\infty} A_{n}=M \cap E$. Since $M \cap E$ is a non-meager set, there is a positive integer $m$ such that $A_{m}$ is dense in some nonempty open subset $U_{0}$ of $X$, i.e., $U_{0} \subseteq \overline{A_{m}}$. By condition (B), it follows that there are nonempty open subsets $G$ of $X$ and $H$ of $Y$ such that $G \times H \subseteq U_{0} \times V_{m}$ and $f(G \times H) \subseteq \overline{f\left(A_{m} \times V_{m}\right)}$. Then, $G \times H \subseteq U_{0} \times V_{m} \subseteq U \times V$ and

$$
\begin{aligned}
& f(G \times H) \subseteq \overline{f\left(A_{m} \times V_{m}\right)} \subseteq \overline{f\left(\bigcup_{x \in A_{m}}\left(\{x\} \times V_{m}\right)\right)} \\
& =\overline{\bigcup_{x \in A_{m}} f\left(\{x\} \times V_{m}\right)}=\overline{\bigcup_{x \in A_{m}} f^{x}\left(V_{m}\right)}
\end{aligned}
$$

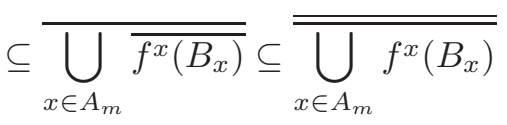

$$
\begin{aligned}
& =\overline{\bigcup_{x \in A_{m}} f^{x}\left(B_{x}\right)}=\overline{f\left(\bigcup_{x \in A_{m}}\left(\{x\} \times B_{x}\right)\right)} \\
& \subseteq \overline{f\left(\bigcup_{x \in M}\left(\{x\} \times B_{x}\right)\right)} \text {. }
\end{aligned}
$$

Hence, by Theorem 5.1, the mapping $f$ is simply continuous. 


\section{SEPARATE AND JOINT PROPERTIES OF POINTWISE DISCONTINUITY}

\section{Jointly mildly continuity}

Theorem 6.1. Let $X$ be a Baire space, $Y$ a space which has a countable pseudo-base, $Z$ a separable metrizable space, $f: X \times Y \rightarrow Z$ a function such that condition $(B)$ holds, and $M=\left\{x \in X: f^{x}\right.$ is mildly continuous $\}$ a residual subset of $X$. Then, $f$ is mildly continuous.

P r o of. Assume the contrary. Do not let $f$ be mildly continuous. This means that $\overline{S(f)} \neq X \times Y$. Then, there are nonempty open subset $U$ of $X$ and $V$ of $Y$ such that $S(f) \cap(U \times V)=\varnothing$. Hence, $U \times V \subseteq((X \times Y) \backslash Q(f)) \cap P(f)$, i.e., $f$ is not quasi-continuous but almost continuous at each point of $U \times V$.

Since $f^{x}$ is mildly continuous for each $x \in U \cap M, \overline{S\left(f^{x}\right)} \supseteq V$. Then, $S\left(f^{x}\right) \cap V \neq \varnothing$ for all $x \in U \cap M$. It means that for each $x \in U \cap M$ there is a point $y_{x} \in V$ such that $f^{x}$ is quasi-continuous at $y_{x}$, or $f^{x}$ is not almost continuous at $y_{x}$.

Considers the sets

$$
A=\left\{x \in U \cap M: y_{x} \in Q\left(f^{x}\right)\right\} \quad \text { and } \quad B=\left\{x \in U \cap M: y_{x} \notin P\left(f^{x}\right)\right\} .
$$

Then, $U \cap M=A \cup B$. Since $X$ is a Baire space, $U \cap M$ is a non-meager subset of $X$. Therefore, at least one of the sets $A$ or $B$ is non-meager. Since $Z$ is a separable metrizable space, $Z$ is a regular second countable space. Let $\left\{V_{n}: n \in \mathbb{N}\right\}$ be a pseudo-base of $Y$ and $\left\{W_{m}: m \in \mathbb{N}\right\}$ a base of $Z$.

First, assume that $A$ is a non-meager subset of $X$. Since for each $x \in A$ the mapping $f$ is not quasi-continuous at $\left(x, y_{x}\right) \in U \times V$, there are open neighborhoods $U(x)$ of $x$ in $X, V(x)$ of $y_{x}$ in $Y$ and a closed neighborhood $W(x)$ of $f\left(x, y_{x}\right)$ in $Z$ such that $U(x) \subseteq U, V(x) \subseteq V$ and $f(G \times H) \nsubseteq \subseteq W(x)$ for arbitrary nonempty open subsets $G$ of $X$ and $H$ of $Y$ such that $G \times H \subseteq U(x) \times V(x)$.

For positive integers $m$ and $n$, we consider the sets

$$
A_{n, m}=\left\{x \in A: f\left(x, y_{x}\right) \in W_{m} \subseteq W(x), V_{n} \subseteq V(x), f^{x}\left(V_{n}\right) \subseteq W_{m}\right\} .
$$

Since, $f^{x}$ is quasi-continuous at $y_{x}$ for each $x \in A, A=\bigcup_{n, m=1}^{\infty} A_{n, m}$. From the fact that $A$ is a meager set, it follows that there are positive integers $n_{0}$ and $m_{0}$ such that $A_{n_{0}, m_{0}}$ is a somewhere dense subset of $X$. Since $f$ satisfies condition (B), there are nonempty open subsets $G$ of $X$ and $H$ of $Y$ such that

$$
G \subseteq \operatorname{int} \overline{A_{n_{0}, m_{0}}}, \quad H \subseteq V_{n_{0}} \quad \text { and } \quad f(G \times H) \subseteq \overline{f\left(A_{n_{0}, m_{0}} \times V_{n}\right)} .
$$

Take any point $a \in A_{n_{0}, m_{0}} \cap G$. Then,

$$
G \cap U(a) \subseteq U(a), \quad H \subseteq V_{n_{0}} \subseteq V(a)
$$

and

$$
f((G \cap U(a)) \times H) \subseteq f(G \times H) \subseteq \overline{f\left(A_{n_{0}, m_{0}} \times V_{n_{0}}\right)} \subseteq \overline{W_{m_{0}}} \subseteq \overline{W(a)}=W(a) .
$$

Since $a \in A_{n_{0}, m_{0}} \subseteq A$, we obtained a contradiction. 


\section{VASYL' NESTERENKO}

Suppose now that $B$ is a non-meager subset of $X$. Since $f$ is almost continuous at a point $\left(x, y_{x}\right) \in U \times V$ for each $x \in B$, for any neighborhood $W$ of $f\left(x, y_{x}\right)$ in $Z$, there are open neighborhoods $U(x, W)$ of $x$ in $X, V(x, W)$ of $y_{x}$ in $Y$, and a subset $O$ of $X \times Y$ with $\bar{O} \supseteq U(x, W) \times V(x, W)$ such that $f(O) \subseteq W$. For positive integers $m$ and $n$, we consider the sets

$$
B_{n, m}=\left\{x \in B: f\left(x, y_{x}\right) \in W_{m}, V_{n} \subseteq V\left(x, W_{m}\right), f^{x}\left(V_{n}\right) \subseteq Z \backslash W_{m}\right\} .
$$

Since, $f^{x}$ is not almost continuous at $y_{x}$ for each $x \in B, B=\bigcup_{n, m=1}^{\infty} B_{n, m}$.

From the fact that $B$ is a meager set, it follows that there are positive integers $n_{0}$ and $m_{0}$ such that $B_{n_{0}, m_{0}}$ is a somewhere dense subset of $X$. Since $f$ satisfies condition (B), there are nonempty open subsets $G$ of $X$ and $H$ of $Y$ such that

$$
G \subseteq i n t \overline{B_{n_{0}, m_{0}}}, \quad H \subseteq V_{n_{0}} \quad \text { and } \quad f(G \times H) \subseteq \overline{f\left(B_{n_{0}, m_{0}} \times V_{n}\right)} .
$$

Take any point $a \in B_{n_{0}, m_{0}} \cap G$. Then, for the neighborhood $U\left(a, W_{m_{0}}\right) \times$ $V\left(a, W_{m_{0}}\right)$ of $\left(a, y_{a}\right)$, there exists a nonempty open set $\left(G \cap U\left(a, W_{m_{0}}\right)\right) \times H \subseteq$ $U\left(a, W_{m_{0}}\right) \times V\left(a, W_{m_{0}}\right)$ such that

$f\left(\left(G \cap U\left(a, W_{m_{0}}\right)\right) \times H\right) \subseteq f(G \times H) \subseteq \overline{f\left(B_{n_{0}, m_{0}} \times V_{n}\right)} \subseteq \overline{Z \backslash W_{m_{0}}}=Z \backslash W_{m_{0}}$.

It means that $f$ is not almost continuous at $\left(a, y_{a}\right) \in U \times V$. This contradiction proves that $B$ is a meager subset of $X$.

Thus, $f$ is mildly continuous.

\section{REFERENCES}

[1] THiElman, H. P.: Types of functions, Amer. Math. Monthly. 60 (1953), 156-161.

[2] KEMPISTY, S.: Sur les fonctions quasicontinues, Fund. Math. 19 (1932), 184-197.

[3] BISWAS, N.: On some mappings in topological spaces, Bull. Calcutta Math. Soc. 61 (1969), 127-135.

[4] BORSÍK, J.-DOBOŠ, J.: On certain decompositions of continuity, Rend. Ist. Matem. Univ. Trieste 20 (1988), 275-282.

[5] PIOTROWSKI, Z.: A survey of results concerning generalized continuity in topological spaces, Acta Math. Univ. Comenianae, 52-53 (1987), 91-110.

[6] NESTERENKO, V.: Joint properties of functions which monotony with respect to the first variable, Mat. Visn. Nauk. Tov. Im. Shevchenka. 6 (2009), 195-201. (In Ukrainian)

[7] GRANDE, Z.: On functions of two variables whose vertical sections have closed graphs, Real Anal. Exchange. 27 (2002), 661-668.

[8] GRANDE, Z.: Quasicontinuity, cliquishness and the Baire property of functions of two variables, Tatra Mt. Math. Publ. 24 (2002), 29-35.

[9] NESTERENKO, V.: On one characterization of joint quasi-continuity, Nauk. Visn. Chernivetskogo Univ., Mat. 336 (2007), 137-141. (In Ukrainian) 


\title{
SEPARATE AND JOINT PROPERTIES OF POINTWISE DISCONTINUITY
}

[10] FUDALI, L. A.: On cliquish functions on product spaces, Math. Slovaca. 33 (1983), 53-58.

[11] NESTERENKO,V.: On symmetrical cliquishness and quasi-continuity of functions of two variables, Topology Appl. (to appear).

[12] MAslyuChenKO, V. K.-MYKhAJLYUK, V. V.-NeSTEREnKO, V. V.: Point discontinuity of multivariable functions, Nauk. Visn. Chernivetskogo Univ., Mat. 111 (2001), 70-76. (In Ukrainian)

[13] MARCUS, S.: Sur les fonctions quasicontinues an sens de S. Kempisty, Colloq. Math. 8 (1961), 47-53.

[14] FROLÍK, Z.: Remarks concerning the invariance of Baire spaces under mappings, Czechoslovak Math. J. 11 (1961), 381-385.

[15] DOnTCHEV, J.-GAnsteR, M.: More on mild continuity, Rend. Ist. Mat. Univ. Trieste. 27 (1995), 47-59.

[16] MASLYUCHENKO, V. K.: Separately Continuous Mappings and Köthe Spaces. Doctoral Thesis (1999). (In Ukrainian)

[17] BORSÍK, J.: Maximal additive and maximal multiplicative family for the class of simply continuous functions, Real Anal. Exchange 20 (1995), 204-211.

[18] BORSÍK, J.-DOBOŠ, J.: On simple continuity points, Real Anal. Exchange. 16 (1991), $552-558$.

[19] MASLYUCHENKO, V. K.-NESTERENKO, V. V.: Joint continuity and quasicontinuity of horizontally quasicontinuous mappings, Ukr. Math. J. 52 (2000), 1952-1955.

Received February 5, 2014

\author{
Chernivtsi National University \\ Department of Mathematical \\ Kotsubinsky Str. 2 \\ $U A-58012$-Chernivtsi \\ UKRAINE \\ E-mail: math.analysis.chnu@gmail.com
}

